\title{
Diversity and ecological characteristics of plants at Sardara banda, village Ikram pur (Baizo kharki) district Mardan, Khyber Pakhtunkhwa (Pakistan)
}

Sajjad Ali ${ }^{*}$, Sami Ullah ${ }^{2}$, Mukhtiar Ali ${ }^{1}$, Wisal Muhammad Khan ${ }^{3}$, Izhar $\mathrm{Ahmad}^{3}$ and Muhammad Hashim $\mathrm{Khan}^{3}$

1. Department of Botany Bacha Khan University, KP, Charsadda-Pakistan

2. Department of Botany, University of Peshawar, Peshawar-Pakistan

3. Department of Botany, Islamia College Peshawar-Pakistan

*Corresponding author's email: Sajjad_ali00@yahoo.com

Citation

Sajjad Ali, Sami Ullah, Mukhtiar Ali, Wisal Muhammad Khan, Izhar Ahmad and Muhammad Hashim Khan.

Diversity and ecological characteristics of plants at Sardara banda, village Ikram pur (Baizo kharki) district Mardan, Khyber Pakhtunkhwa (Pakistan). Pure and Applied Biology. Vol. 5, Issue 4, pp1271-1277. http://dx.doi.org/10.19045/bspab.2016.50152

Received: 12/09/2016 Revised: 03/11/2016

Accepted: 10/11/2016

Online First: 18/11/2016

\section{Abstract}

Floral diversity at Ikram pur (Baizo kharki) district Mardan comprised a total of 68 plant species belonging to 63 genera and 34 families. The leading families were Poaceae and Asteraceae having 06 species each $(8.82 \%)$, Lamiaceae with 05 species $(7.35 \%)$, followed by Solanaceae and Moraceae having 04 species each (5.88\%), Mimosaceae and Apocynaceae having 3 species each (4.41\%) while Asclepiadaceae, Chenopodiaceae, Brassicaceae, Zygophyllaceae, Caryophyllaceae, Rhamnaceae, Malvaceae, Euphorbiaceae, Papilionaceae and Myrtaceae having 2 species each (2.94\%). Rest of 17 families shared 01 species each (1.47\%). The dominant life form was therophyte with 20 species $(29.41 \%)$, followed by nanophanerophyte having 16 species (23.52\%), microphanerophyte was represented by 13 species $(19.11 \%)$, hemicryptophyte 9 species $(13.23 \%)$, geophytes 6 species $(8.82 \%)$ and chamaephytes 4 species $(5.88 \%)$. Dominant leaf size was nanophylls with 36 species (52.94\%), followed by microphylls 12 species (17.64\%), leptophylls 8 species (11.76\%), mesophylls 6 species $(8.82 \%)$, megaphylls 4 species $(5.88 \%)$ and aphyllous by 2 species $(2.94 \%)$. Fifty five plant species $(80.88 \%)$ were of dry habitate, followed by 8 species $(11.76 \%)$ in wet and 6 species $(8.82 \%)$ both dry and wet habitats. In the 68 plants species, 57 were non-spiny and 11 spiny.

Keywords: Ecological charcteristics; Baizo Kharki; Plant diversity

Introduction

Area introduction (Ikram Pur)

\section{Location}

Ikram Pur is also represented with the name

Baizo Kharki. Ikram Pur situated in $34^{\circ} .4966736$ latitude and $72^{\circ} .0109411$ to the longitude. It is located in north east belt of the Mardan. On its boundary Malakand Agency is located. Maximum rain occurred during "July and August". 


\section{Flora}

The flora of the Ikram pur areas are rich. The most common trees in the area are Acasia modesta, Acasia nilotica, Morus alba, Morus nigra, Eucalyptus camaldulensis, Populus indica, Ziziphus jujuba, Malia azedarach and Dalbergia sissoo. Shrubs are Dodonia viscose, Datura alba, Justicia adhatoda, and the most common herbs are Cynodon dactylon, Cymbopogon distans, Fumaria indica, Boerhavia diffusa, Cyperus rotundus, Chenopodium album, Tribulus terrestris and Chenopodium murale etc.

\section{Introduction floristic diversity}

Information concerning with floristic composition of a region is perquisite for any region. Flora composition reflects the variety of plants in a area which has been affected by many factors like overgrazing, soil composition, plant cutting. Plants of an area give a detailed account for the environmental conditions of the specific area [1].

Floral record gives us a lot of information about the area like the relation of indigenous to the plant [2]. Ecologists at all times effort to know the variance in types of variety along with environmental change like altitudinal slope in mountainous biomes [1, 3]. For understanding the relationship of living and non-living component in a ecosystem need to study the diversity of an area [4]. Flora oftenly the source for botanical information about a specific area for a proper studies [5].

Categorization of natural ecosystems into possible plant communities and habitat types is important for the long term managing of natural resources [6]. The coastal plants develop morphological, physiological and metabolic character not only to grow but also complete their life cycle under salt stressed condition $[7,8]$.

\section{Material and methods}

\section{Collection and identification}

Survey was carried out in many spells, which was arranged in different seasons from the village for the purpose to collect the plant materials. After collection local and botanical name, family name, seasonal availability, habit, habitat, life form, leaf size, and Spinacious characters of plant species were recorded.

\section{Preservation of plants}

After collection and identification plant materials was appropriately pressed, dry out and well-kept rendering the standard method. Old newspapers were used along with naphthalene to protect from the fungal attack. Plants were accurately fixed on herbarium sheets and submitted to herbarium of Bacha Khan University, Charsadda, Khyber Pakhtunkhwa, Pakistan for further record.

\section{Results and discussion}

\section{Distribution of families}

Floral diversity comprised of 68 plant species belonging to 63 taxa and 34 families. Asteraceae and Poaceae were the dominant families having 06 species each $(8.82 \%)$, followed by Lamiaceae with 05 species $(7.35 \%)$, Moraceae and Solanaceae having 04 species each (5.88\%), Apocynaceae, Mimosaceae having 3 species each (4.41\%), Asclepiadaceae, Chenopodiaceae, Brassicaceae, Myrtaceae, Malvaceae, Papilionaceae, Rhamnaceae, Euphorbiaceae and Zygophyllaceae, having 02 species each $(2.94 \%)$. The rest of 17 families contributed 01 species each $(1.47 \%)$. Total of 57 plant species $(83.82 \%)$ were spiny and 11 $(16.17 \%)$ were non-spiny. The herbs were found as the common fraction in the studied area represented by 40 species $(58.82 \%)$, followed by trees and shrubs represented by 14 species each (20.58\%). 55 species $(80.88 \%)$ have dry habitat, followed by wet habitat having 8 plant species $(11.76 \%), 5$ 
species $(7.35 \%)$ is recorded in both conditions. Poaceae and Asteraceae were the leading families [9]. [10-12] also observed that these families were leading in their individual study areas. From some other part of Pakistan the same study was noted [13].

\section{Life form}

The dominant class was therophyte having 20 species (29.41\%), followed by nanophanerophyte having 16 species (23.52\%), microphanerophyte represented by 13 species (19.11\%), hemicryptophyte 9 species $(13.23 \%)$, geophytes represented by 6 species $(8.82 \%)$ and chamaephytes 4 species $(5.88 \%)$. According to Shah et al. [23] the dominance of therophyte reflects an effective strategy for avoiding water losses due to moisture extremes and water insufficiency. Therophytes and chamaeophytes were the dominant life form in the areas where rainfall was less [14]. According to study of Nadaf and Murtazvi [21] hemicryptophyte was the dominant life form belong to Irano-Turanian. In south eastern Brazil the Therophytic and phanerophytic vegetation were in common $[15,16]$.

\section{Leaf size}

The most dominant leaf size was nanophyll with 36 species $(52.94 \%)$, followed by microphyll 12 species (17.64\%), leptophyll
8 species $(11.76 \%)$ and mesophyll 6 species (8.82\%), megaphyll 4 species $(5.88 \%)$ and aphyllous 2 species (2.94\%). Leaf size class showed changes from season to season, while evergreen and perennials retain it. [17] observed that leaf size completely related to deficiency in the soil nutrients. [17, 18] reported microphyllous and nanopyllous as the leading leaf size from Kotli Azad Kashmir and Waziristan. Leaf spectra of plants consisted of microphyll followed by mesophyll and nanophyll from their study area [19, 20]. The results "of Kar et al. [13] are also in line with our result as they reported the dominance of therophyte and nanophanerophytes in grasslands of Odisha, India" [21].

\section{Seasonal variation}

Seasonal availability show that 54 plant species $(79.41 \%)$ were present in spring, followed by 46 species $(67.64 \%)$ were present in winter, 44 plant species $(64.70 \%)$ were present in summer season and 32 plant species $(47.05 \%)$ were present in autumn. In maximum of the season the therophytic and nanophanerophytic vegetation were dominant but in spring this case is very common [22]. Dominancy of therophytes occurs due to uncomplimentary environmental form as suggested by others [22-26], which is in conformity to our work.

Table 1. Floristic list of Sardara Banda, Ikram pur (Baizo kharki) Mardan

\begin{tabular}{|l|l|l|l|l|l|l|l|l|l|l|l|l|}
\hline $\begin{array}{l}\text { S. } \\
\text { No }\end{array}$ & $\begin{array}{l}\text { "Plant Botanical } \\
\text { name" }\end{array}$ & Family name & Local name & $\mathbf{A}$ & $\mathbf{W}$ & $\mathbf{S P}$ & $\mathbf{S M}$ & $\begin{array}{l}\text { Life } \\
\text { form }\end{array}$ & $\begin{array}{l}\text { Leaf } \\
\text { size }\end{array}$ & Habitat & Habit & Spinacious \\
\hline 1 & Justicia adhatoda L. & Acanthaceae & Baikerh & + & + & + & + & NP & L & D & SB & - \\
\hline 2 & $\begin{array}{l}\text { Amaranthus viridis } \\
\text { L. }\end{array}$ & Amaranthaceae & Ganhar & - & + & + & + & TH & N & D & H \\
\hline 3 & Nerium olender L. & Apocynaceae & Ganderay & + & + & + & + & NP & MIC & D & SB & - \\
\hline 4 & $\begin{array}{l}\text { Dodonaea viscosa } \\
\text { (L.) Jacq. }\end{array}$ & Apocynaceae & Ghawaraskay & + & + & + & + & NP & N & D & SB \\
\hline 5 & $\begin{array}{l}\text { Caralluma edulis } \\
\text { Edgew. Benth. ex } \\
\text { Hook.f. }\end{array}$ & Apocynaceae & Pamunkay & - & + & - & + & G & AP & D & H \\
\hline 6 & $\begin{array}{l}\text { Calotropis prosera } \\
\text { Acit. }\end{array}$ & Asclepiadaceae & Spalmay & + & + & + & + & CH & MES & D & SB \\
\hline 7 & $\begin{array}{l}\text { Periploca aphylla } \\
\text { Decne. }\end{array}$ & Asclepiadaceae & Barrhara & + & + & + & + & NP & AP & D & SB \\
\hline 8 & $\begin{array}{l}\text { Asparagus } \\
\text { officinalis L. }\end{array}$ & Asparagaceae & Tindonray & - & - & - & + & CH & L & D & SB \\
\hline 9 & Xanthium & Asteraceae & Gishkay & - & - & + & - & TH & N & D & H & + \\
\hline
\end{tabular}


Ali et al.

\begin{tabular}{|c|c|c|c|c|c|c|c|c|c|c|c|c|}
\hline & strumarium $\mathrm{L}$. & & & & & & & & & & & \\
\hline 10 & Sonchus asper L. & Asteraceae & Shodapai & _ & + & - & - & $\mathrm{TH}$ & MIC & $\mathrm{W}$ & $\mathrm{H}$ & \\
\hline 11 & $\begin{array}{l}\text { Parthenium } \\
\text { hysterophorus } \mathrm{L} .\end{array}$ & Asteraceae & $\begin{array}{l}\text { Lewanai } \\
\text { Bang }\end{array}$ & + & - & + & + & MP & MIC & $\mathrm{D} \& \mathrm{~W}$ & $\mathrm{H}$ & - \\
\hline 12 & $\begin{array}{l}\text { Silybum marianum } \\
\text { (L.) Geartn. }\end{array}$ & Asteraceae & $\begin{array}{l}\text { Mulla } \\
\text { charchugh }\end{array}$ & - & - & + & - & $\mathrm{TH}$ & MEP & $\mathrm{D}$ & $\mathrm{H}$ & + \\
\hline 13 & $\begin{array}{l}\text { Calendula arvensis } \\
\text { M.Bieb. }\end{array}$ & Asteraceae & Zyar gulle & + & - & + & - & $\mathrm{TH}$ & $\mathrm{N}$ & $\mathrm{D}$ & $\mathrm{H}$ & - \\
\hline 14 & $\begin{array}{l}\text { Echinops echinotus } \\
\text { Roxb. }\end{array}$ & Asteraceae & Kariza & - & + & - & + & NP & MIC & $\mathrm{D}$ & SB & + \\
\hline 15 & $\begin{array}{l}\text { Heliotropium } \\
\text { europaeum L. }\end{array}$ & Boraginaceae & Spen guley & - & - & + & - & $\mathrm{TH}$ & MIC & $\mathrm{D}$ & $\mathrm{H}$ & - \\
\hline 16 & $\begin{array}{l}\text { Coronopus didymus } \\
\text { (L). Sm. }\end{array}$ & Brassicaceae & Ganda botay & - & + & + & + & $\mathrm{TH}$ & MIC & $\mathrm{D}$ & $\mathrm{H}$ & - \\
\hline 17 & Eruca sativa Mill. & Brassicaceae & Jumama & _- & + & + & _- & NP & MIC & $\mathrm{D}$ & $\mathrm{H}$ & _ \\
\hline 18 & Cannabis sativa $\mathrm{L}$. & Canabaceae & Bhang & _- & + & + & + & MP & $\mathrm{N}$ & W\&D & $\mathrm{H}$ & _ \\
\hline 19 & $\begin{array}{l}\text { Stellaria media (L.) } \\
\text { Vill. }\end{array}$ & Caryophyllaceae & Not known & - & - & - & + & $\mathrm{TH}$ & $\mathrm{N}$ & $\mathrm{D}$ & $\mathrm{H}$ & - \\
\hline 20 & Silene conidia $\mathrm{L}$. & Caryophyllaceae & Mangotay & _- & + & + & + & NP & $\mathrm{N}$ & $\mathrm{W}$ & $\mathrm{H}$ & _ \\
\hline 21 & $\begin{array}{l}\text { Chenopodium album } \\
\mathrm{L}\end{array}$ & Chenopodiaceae & Chalwaye & - & + & + & - & TH & $\mathrm{N}$ & $\mathrm{D}$ & $\mathrm{H}$ & - \\
\hline 22 & C. murale $\mathrm{L}$. & Chenopodiaceae & Sarmey & _- & + & + & _. & $\mathrm{TH}$ & $\mathrm{L}$ & $\mathrm{D}$ & $\mathrm{H}$ & _ \\
\hline 23 & $\begin{array}{l}\text { Convolvulus } \\
\text { arvensis L. }\end{array}$ & Convolulaceae & Perwatye & - & + & - & - & $\mathrm{TH}$ & $\mathrm{N}$ & $\mathrm{D}$ & $\mathrm{H}$ & - \\
\hline 24 & $\begin{array}{l}\text { Ricinus communies } \\
\text { L. }\end{array}$ & Euphorbiaceae & Aranda & + & + & + & + & $\mathrm{CH}$ & MEP & $\mathrm{D}$ & SB & - \\
\hline 25 & $\begin{array}{l}\text { Euphorbia } \\
\text { helioscopia } \mathrm{L} .\end{array}$ & Euphorbiaceae & Mandanro & - & - & + & - & $\mathrm{TH}$ & $\mathrm{N}$ & $\mathrm{D}$ & $\mathrm{H}$ & - \\
\hline 26 & Lathyrus aphaca L. & Fabaceae & Chelu & + & _ & + & _- & $\mathrm{TH}$ & $\mathrm{N}$ & $\mathrm{W}$ & $\mathrm{H}$ & _ \\
\hline 27 & $\begin{array}{l}\text { Fumaria indica } \\
\text { (Hausskn.) Pugsley }\end{array}$ & Fumaraceae & Papra & - & + & - & - & $\mathrm{TH}$ & $\mathrm{N}$ & $\mathrm{D}$ & $\mathrm{H}$ & - \\
\hline 28 & $\begin{array}{l}\text { Mentha longifolia } \\
\text { (L.) L. }\end{array}$ & Lamiaceae & Velanay & - & + & + & - & G & $\mathrm{N}$ & W & $\mathrm{H}$ & - \\
\hline 29 & Mentha spicata $\mathrm{L}$. & Lamiaceae & Podina & - & + & + & + & $\mathrm{G}$ & $\mathrm{N}$ & W & $\mathrm{H}$ & _ \\
\hline 30 & $\begin{array}{l}\text { Salvia } \\
\text { moorcroftiana Wall. } \\
\text { ex Benth. }\end{array}$ & Lamiaceae & Khar Dug & - & - & - & + & NP & MES & $\mathrm{D}$ & $\mathrm{H}$ & - \\
\hline 31 & $\begin{array}{l}\text { Otostegia limbata } \\
\text { (Benth.) Boiss. }\end{array}$ & Lamiaceae & Spen Azghay & + & + & + & + & NP & $\mathrm{N}$ & $\mathrm{D}$ & SB & + \\
\hline 32 & $\begin{array}{l}\text { Colebrookea } \\
\text { oppositifolia } \mathrm{Sm} .\end{array}$ & Lamiaceae & $\begin{array}{l}\text { Zangali } \\
\text { Baikerh } \\
\end{array}$ & + & + & + & + & $\mathrm{NP}$ & $\mathrm{L}$ & D & SB & - \\
\hline 33 & $\begin{array}{l}\text { Dalbergia sisso } \\
\text { Roxb. }\end{array}$ & Leguminosae & Shawa & + & + & + & + & MP & $\mathrm{N}$ & $\mathrm{W} \& \mathrm{D}$ & $\mathrm{T}$ & - \\
\hline 34 & $\begin{array}{l}\text { Grewia optiva } \\
\text { J.R.Drumm. ex } \\
\text { Burret }\end{array}$ & Malvaceae & Pastawoony & + & + & + & + & NP & $\mathrm{N}$ & $\mathrm{D}$ & $\mathrm{T}$ & - \\
\hline 35 & $\begin{array}{l}\text { Malva neglecta } \\
\text { Wallr. }\end{array}$ & Malvaceae & Parenak & - & - & + & + & $\mathrm{H}$ & $\mathrm{N}$ & D & $\mathrm{H}$ & - \\
\hline 36 & Melia azedarech $\mathrm{L}$. & Meliaceae & Tora Shandai & + & + & + & + & MP & $\mathrm{N}$ & $\mathrm{D}$ & $\mathrm{T}$ & _ \\
\hline 37 & $\begin{array}{l}\text { Albizia lebbek (L.) } \\
\text { Benth. }\end{array}$ & Mimosaceae & Sareekh & + & + & + & + & MP & $\mathrm{N}$ & D & $\mathrm{T}$ & - \\
\hline 38 & $\begin{array}{l}\text { Acacia modista } \\
\text { Wall. }\end{array}$ & Mimosaceae & Paloosa & + & + & + & + & MP & $\mathrm{L}$ & D & $\mathrm{T}$ & + \\
\hline 39 & $\begin{array}{l}\text { Acacia nilotica }(\mathrm{L} .) \\
\text { Delile }\end{array}$ & Mimosaceae & Keekar & + & + & + & + & MP & $\mathrm{L}$ & D & $\mathrm{T}$ & + \\
\hline 40 & $\begin{array}{l}\text { Broussonetia } \\
\text { papyrifera }(\mathrm{L} .) \mathrm{L}\end{array}$ & Moraceae & Gultoot & + & + & + & + & MP & MIC & $\mathrm{D}$ & $\mathrm{T}$ & - \\
\hline 41 & Morus alba L. & Moraceae & Toot & + & + & + & + & MP & MES & $\mathrm{D}$ & $\mathrm{T}$ & \\
\hline 42 & Ficus carica $\mathrm{L}$. & Moraceae & Inzar & + & + & + & + & NP & MES & $\mathrm{D}$ & $\mathrm{T}$ & \\
\hline 43 & Morus nigra L. & Moraceae & Toor toot & + & + & + & + & MP & MES & $\mathrm{D}$ & $\mathrm{T}$ & \\
\hline
\end{tabular}




\begin{tabular}{|c|c|c|c|c|c|c|c|c|c|c|c|c|}
\hline 44 & Populus indica L. & Myrtaceae & Sufeedad & + & + & + & + & MP & MES & $\mathrm{D}$ & $\mathrm{T}$ & 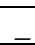 \\
\hline 45 & $\begin{array}{l}\text { Eucalyptus } \\
\text { camaldulensis } \\
\text { Dehnh. }\end{array}$ & Myrtaceae & Lachi & + & + & + & + & MP & $\mathrm{N}$ & $\mathrm{D}$ & $\mathrm{T}$ & - \\
\hline 46 & Boerhavia diffusa $\mathrm{L}$. & Nyctaginaceae & Ensut & - & + & _ & _ & $\mathrm{H}$ & $\mathrm{N}$ & $\mathrm{D}$ & $\mathrm{H}$ & - \\
\hline 47 & $\begin{array}{l}\text { Trifolium } \\
\text { alexendrianum } \mathrm{L} .\end{array}$ & Papilionaceae & Shotal & - & + & + & - & $\mathrm{TH}$ & $\mathrm{N}$ & $\mathrm{W}$ & $\mathrm{H}$ & - \\
\hline 48 & $\begin{array}{l}\text { Medicago polyorpha } \\
\text { L. }\end{array}$ & Papilionaceae & Peeshtare & - & - & + & - & $\mathrm{TH}$ & $\mathrm{N}$ & $\mathrm{D}$ & $\mathrm{H}$ & - \\
\hline 49 & $\begin{array}{l}\text { Cynodon dactylon } \\
\text { (L.) Pers. }\end{array}$ & Poaceae & Kabal & + & + & + & + & $\mathrm{H}$ & $\mathrm{N}$ & W\&D & $\mathrm{H}$ & - \\
\hline 50 & $\begin{array}{l}\text { Cymbopogon distans } \\
\text { (Nees ex Steud.) W. } \\
\text { Watson }\end{array}$ & Poaceae & Sargaray & - & - & + & - & $\mathrm{H}$ & $\mathrm{N}$ & $\mathrm{D}$ & $\mathrm{H}$ & - \\
\hline 51 & Cyperus rotundus $\mathrm{L}$. & Poaceae & Dela & - & + & + & + & G & $\mathrm{N}$ & $\mathrm{W}$ & $\mathrm{H}$ & - \\
\hline 52 & Avena sativa $\mathrm{L}$. & Poaceae & Jamder & _ & + & + & $=$ & $\mathrm{H}$ & $\mathrm{N}$ & $\mathrm{D}$ & $\mathrm{H}$ & _ \\
\hline 53 & $\begin{array}{l}\text { Desmostachya } \\
\text { bipinnata (L.) Stapf. }\end{array}$ & Poaceae & drab & + & - & + & + & $\mathrm{H}$ & $\mathrm{N}$ & W\&D & $\mathrm{H}$ & - \\
\hline 54 & Apluda mutica $\mathrm{L}$. & Poaceae & Wahoo & + & + & + & + & NP & MIC & $\mathrm{D}$ & $\mathrm{H}$ & _ \\
\hline 55 & Rumex dentatus L. & Polygonaceae & Shalkhy & __ & - & + & - & $\mathrm{G}$ & MEP & $\mathrm{W}$ & $\mathrm{H}$ & - \\
\hline 56 & $\begin{array}{l}\text { Portulaca oleracea } \\
\text { L. }\end{array}$ & Portulaceae & Warharay & - & + & - & - & G & $\mathrm{N}$ & $\mathrm{D}$ & $\mathrm{H}$ & - \\
\hline 57 & $\begin{array}{l}\text { Anagallis arvensis } \\
\text { L. }\end{array}$ & Primulaceae & $\begin{array}{l}\text { Ghamay } \\
\text { gulay }\end{array}$ & - & - & - & + & $\mathrm{TH}$ & $\mathrm{N}$ & $\mathrm{D}$ & $\mathrm{H}$ & - \\
\hline 58 & $\begin{array}{l}\text { Sageretia thea } \\
\text { (Osbeck).M.C. }\end{array}$ & Rhamnaceae & Mamanra & - & + & - & + & $\mathrm{NP}$ & $\mathrm{N}$ & $\mathrm{D}$ & SB & - \\
\hline 59 & Ziziphus jujuba Mill. & Rhamnaceae & Baira & + & + & + & + & NP & $\mathrm{N}$ & $\mathrm{D}$ & $\mathrm{T}$ & + \\
\hline 60 & $\begin{array}{l}\text { Monotheca buxifolia } \\
\text { (Falc.) A. DC. }\end{array}$ & Sapotaceae & Gurgura & + & + & + & + & NP & $\mathrm{N}$ & $\mathrm{D}$ & SB & + \\
\hline 61 & $\begin{array}{l}\text { Verbascum thapsus } \\
\text { L. }\end{array}$ & $\begin{array}{l}\text { Scrophulariacea } \\
\mathrm{e}\end{array}$ & Khar Ghawag & - & - & + & - & $\mathrm{TH}$ & MEP & $\mathrm{D}$ & $\mathrm{H}$ & - \\
\hline 62 & $\begin{array}{l}\text { Ailanthus altissima } \\
\text { SW. }\end{array}$ & Simaroubaceae & Dasi Shandai & + & + & + & + & MP & $\mathrm{N}$ & $\mathrm{D}$ & $\mathrm{T}$ & - \\
\hline 63 & $\begin{array}{l}\text { Datura alba Rumph } \\
\text { ex Nees. }\end{array}$ & Solanaceae & Batora & - & - & + & - & $\mathrm{TH}$ & MIC & $\mathrm{D}$ & $\mathrm{H}$ & - \\
\hline 64 & $\begin{array}{l}\text { Solanum surratense } \\
\text { Burm.f. }\end{array}$ & Solanaceae & Maraghonay & + & - & - & - & $\mathrm{H}$ & $\mathrm{N}$ & $\mathrm{D}$ & $\mathrm{H}$ & + \\
\hline 65 & $\begin{array}{l}\text { Withania somnifera } \\
\text { (L.) Dunal }\end{array}$ & Solanaceae & Koti lal & - & - & + & + & $\mathrm{CH}$ & MIC & $\mathrm{D}$ & $\mathrm{H}$ & - \\
\hline 66 & Solanum nigrum L. & Solanaceae & Kach machu & $\ldots$ & _ & + & + & $\mathrm{TH}$ & MIC & $\mathrm{D}$ & $\mathrm{H}$ & _ \\
\hline 67 & Tribulus terrestris L. & Zygophyllaceae & Merkundai & + & - & + & - & $\mathrm{H}$ & $\mathrm{L}$ & $\mathrm{D}$ & SB & + \\
\hline 68 & Fagonia arabica $\mathrm{L}$. & Zygophyllaceae & Azghakey & + & + & $\ldots$ & + & $\mathrm{H}$ & $\mathrm{L}$ & $\mathrm{D}$ & SB & + \\
\hline
\end{tabular}

Seasonal availability: Autumn $=\mathrm{A}$, Winter $=\mathrm{W}$, Summer $=$ SM, Spring $=$ SP.

Life form: Therophyte $=\mathrm{TH}$, Hemicryptophyte $=\mathrm{H}$, Chamaephytes $=\mathrm{CH}$, Nanophenerophyte $=\mathrm{NP}$,

Geophytes =G, Microphanerophyte $=$ MP

Leaf size: Nanophyll = N, Leptophyll = L, Microphyll = MIC, Mesophyll = M, Megaphyll = MEP, Aphyllous = AP,

Habitat: Dry $=$ D, Wet $=$ W

Habit: Herbs $=\mathrm{H}$, Shrubs $=\mathrm{SB}$, Trees $=\mathrm{T}$

\section{Conclusions}

Species diversity and genetic diversity enhance largely biodiversity of an ecosystem. The study area is floristically rich which intrinsically determine the human role both as aesthetic and utilitarian component in maintaining and creating biodiversity.

\section{Authors' contributions}

Conceived and designed the experiments: S Ali \& S Ullah, Performed the experiments: M Ali \& S Ali, Analyzed the data Identification of Plants: WM Khan, I Ahmad 
\& MH Khan, Contributed reagents/ materials/ analysis tools: S Ali \& S Ullah, Wrote the paper: S Ali.

\section{References}

1.Ali SI (2008). The significance of flora with special reference to Pakistan. Pak. J. Bot 40(30): 967-971.

2.Ali SI \& Qaisar M (1995-2009). Flora of Pakistan. Pakistan Agricultural Research Council, Islamabad.

3.Badshah L, Hussain F \& Akhtar N (2010). Vegetation structure of subtropical forest of Tabai, South Waziristan, Pakistan. Front. Agri. China 4(2): 232-236.

4.Badshah L, Hussain F \& Sher Z (2013). Floristic inventory, ecological characteristics and biological spectrum of rangeland, district tank, pakistan. Pak. J. Bot 45(4): 1159-1168.

5.Batalha MA \& Martins FR (2002). Lifeform spectra of Brazilian Cerrado sites. Flora, Morphology, Distribution, Functional Ecology of Plants 197(6): 452-460.

6.Batalha MA \& Martins FR (2004). Floristic frequency, and vegetation life form spectra of a Cerrado site. Braz. $J$. Biol 64(2): 203-209.

7.Chah K, Eze F, Emuelosi CA \& Esimone CO (2006). Antibacterial and wound healing properties of methanolicextracts of some Nigerian medicinal plants .J.Ethnopharm 104: $164-167$.

8.Chopra RN, Nayar SL \& Chopra IC (2006). Glossary of Indian Medicinal Plants. National Institute of Science Communication and Information Resources, New Delhi.80.

9.Costa RC, Araujo FS \& Lima-Verde LW (2007). Flora and life-form spectrum in an area of deciduous thorn woodland (Caatinga) in Northeastern, Brazil. $J$. Arid Environments 68(2): 237-247.

10. Daubenmire RF (1968). Plant Communities: A textbook of Plant
Synecology. Harper \& Row, New York.

11. Durrani MJ, Hussain F \& Rehman SU (2005). Ecological characteristics of plants of Harboi rangeland, Kalat, Pakistan. J. Trop. \& Sub Trop. Bot 13(2): 130-138.

12. Guo QS, Wang XF, Bar G, Kang Y, Hong M, Pei SX \& Zhang FJ (2009). Life form spectra, leaf character, and hierarchical-synusia structure of vascular plants in Thuja sutchuehensis community. Pub. Med 20(9): 2057-2062.

13. Kar PK, Biswal AK \& Barik KL (2010). Floristic composition and biological spectrum of a grassland community of Rangamatia in the district of Mayurbhanj, Odisha. J. Curr. Sci 15(2): 465-469.

14. Keith DA (1988). Floristic lists of New South Wales (III). Cunninghamia 2:3973.

15. Khan MA \& Qaiser M (2006). Halophytes of Pakistan: Characteristics, Distribution and Potential Economic usages. In: Sabkha Ecosystems. (Eds.): M.A. Khan, H. Barth. G.C. Kust and B. Boer Springer, The Netherlands. pp. 129-153.

16. Malik NZ \& Malik ZH (2004). Present status of subtropical chirpine vegetation of Kotli Hills, Azad Jammu and Kashmir. J. Res. Sci 15(1): 85-90.

17. Malik ZH, Hussain F \& Malik NZ (2007). Life form and leaf size spectra of plant communities Harbouring Ganga Chotti and Bedori Hills during (19992000). Int. J. Agri \& Bio., 9(6): 833838.

18. Manhas RK, Singh L, Vasistha HB \& Negi M (2010). Floristic diversity of protected ecosystems of Kandi Region of Punjab, India. New York Sci. J 3(4): 96-103.

19. Marwat Q \& Qureshi RA (2000). A checklist of the vascular plants found in 
upper Siran reserved and guzara forests, District Manshera, Pakistan. Pak. J. Pl. Sci 6(1-2): 43-57.

20. Musila WM, Kinyamario JI \& Jungerius PD (2003). Vegetation dynamics of coastal sand dunes near Malindi, Kenya. Afri. J. Eco 39(2): 170-177.

21. Nadaf M \& Mortazavi SM (2011). Investigation flora and lif form of plants in protected region Sarigol, North Khrasan Province, Iran. Pak. J. Boil. Sci 14(1): $\quad$ 78-81.

22. Qureshi R, Maqsood M, Arshad M \& Chaudhry AK (2011). Ethno medicinal uses of plants by the people of kadhi areas of Khushab, Punjab, Pakistan. Pak. J. Bot 43(1): 121-133.

23. Shah M, Hussain F, Shah SNM, Ahmad I \& Wasila H (2013). Life form and Floristic Characteristics Along
Altitudinal Gradient of Humid Temperate Forests Located in Remote Area of Pakistan, Global J. of Biodi. Sci, and Manag 3(2): 276-281, ISSN 20740875

24. Sher Z \& Khan ZU (2007). Floristic composition, life form and leaf spectra of the vegetation of Chagharzai Valley, District Buner. Pak. J. Pl. Sci 13(1): 5766.

25. Tavili A \& Jafari M (2009). Interrelations between plants and environmental variables. Int. $J$. Environ. Res 3(2): 239-246.

26. Vetaas \& Grytnes J (2002). Distribution of vascular plant species richness and endemic richness along the Himalayan elevation gradient in Nepal. Global Ecol. Biogeography 11(4):291- 301. 